\title{
CONCENTRACIÓN INDUSTRIAL, TAMAÑO \\ DE LA EMPRESA Y PRODUCTIVIDAD DE LOS FACTORES EN MÉXICO*
}

\author{
Manuel Gollás \\ El Colegio de México
}

\section{INTRODUCCIÓN}

El SECTOR INDUSTRIAL de la economía mexicana muestra un grado elevado de concentración. En 1970 la distribución del empleo y la producción por empresa según su tamaño, muestra que las pequeñas (con menos de cinco trabajadores), que representaban el $63 \%$ de las empresas industriales, produjeron sólo el $2.4 \%$ del producto de la rama (véase el

Cuadro 1

MÉxico: NÚMERo de ESTABLECIMIENTOS, EMPLEO Y VALOR DE LA PRODUCCIÓN DE EMPRESAS INDUSTRIALES POR CLASES DE TAMAÑo, 1970

(Porcientos)

\begin{tabular}{|c|c|c|c|c|}
\hline \multicolumn{2}{|c|}{$\begin{array}{l}\text { Tamaño de } 10 \\
\text { empresa a }\end{array}$} & $\begin{array}{l}\text { Proporción respecto } \\
\text { del total de } \\
\text { empresas }\end{array}$ & $\begin{array}{l}\text { Proporcion respecto } \\
\text { del empleo total }\end{array}$ & $\begin{array}{c}\text { Proporcion respecto } \\
\text { de la produccion } \\
\text { total }\end{array}$ \\
\hline 0 & 5 & 62.86 & 7.2 & 2.4 \\
\hline 6 & 15 & 17.56 & 6.6 & 3.4 \\
\hline 16 & -25 & 5.33 & 4.4 & 3.3 \\
\hline 26 & -50 & 5.65 & 8.5 & 6.5 \\
\hline 51 & $-\quad 75$ & 2.54 & 6.5 & 5.7 \\
\hline 76 & -100 & 1.46 & 5.4 & 5.1 \\
\hline 101 & - 175 & 1.95 & 10.9 & 10.9 \\
\hline 176 & -250 & 0.93 & 8.2 & 9.0 \\
\hline 251 & -350 & 0.59 & $(7.3$ & 8.1 \\
\hline 351 & -500 & $1.7, \quad 0.46$ & 8.0 & $5 3 . 7 \longdiv { 9 . 8 }$ \\
\hline 501 & .750 & 1.730 .32 & $\{8.1$ & $53.7\{10.7$ \\
\hline $75 \mathrm{~L}$ & - & 10.34 & 18.9 & 25.1 \\
\hline Tota & & 100 & 100 & 100 \\
\hline
\end{tabular}

FUENTE: Manuel Gollás, "Reflexiones sobre la concentración económica y el crecimiento de las empresas", El Trimestre Económico, Núm. 166, Vol. XLII (2), México, abril-junio de 1976, pp. 457-485.

a Según el número de trabajadores.

* Este artículo fue publicado originalmente como el Discussion Paper, Núm. 293, del Economic Growth Center, Yale University, durante la permanencia del autor en 1968 como profesor visitante en esa Universidad. 
Cuadro 2

MÉXICo: INDICES DE CONCENTRACIÓN INDUSTRIAL SEGÚN EL NÚMERO DE TRA BAJADORES

$1965-1970$

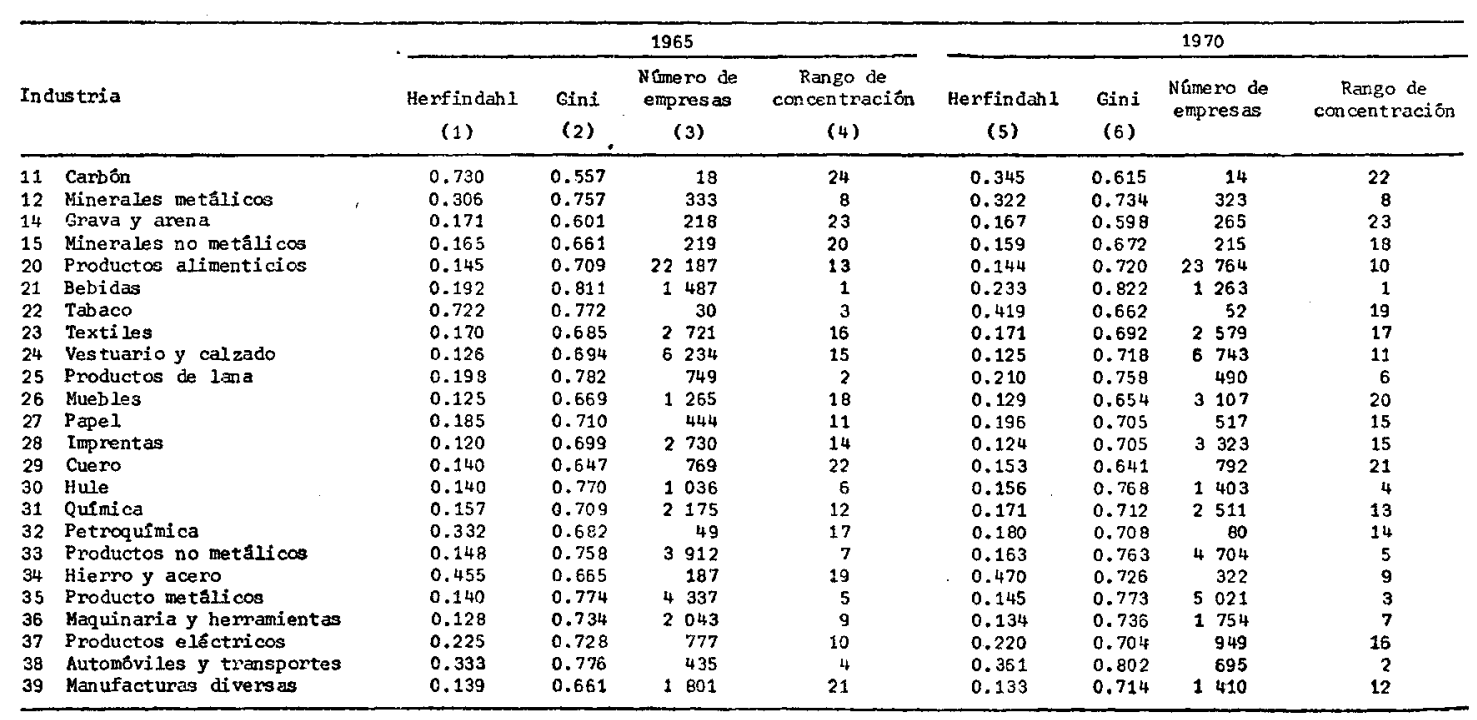

FUENTE: Manuel Gollas, "Reflexiones sobre la concentración económica y el crecimiento de las empresas”, El Trimestre Económico, Núm. 166, Vol. XLII (2), México, abril-junio de 1976, pp. 457-485. 
cuadro 1). Por otro lado, un número reducido de las empresas "grandes" (250 o más trabajadores) las cuales constituían sólo el 1.7\% del número total de empresas, generaban cerca del $54 \%$ del producto industrial y ocupaban alrededor del $42 \%$ de la fuerza de trabajo en el sector.

Existen diversos índices para medir el grado de concentración industrial. Entre otros cabe mencionar el coeficiente del Gini, el índice de Herfindahal, el número de empresas que en conjunto producen el $\mathbf{8 0} \%$ de la producción industrial y, el número de empresas que en conjunto dan empleo al $80 \%$ de la fuerza de trabajo industrial. Los índices de concentración industrial de Gini y de Herfindahal para México se mues. tran en el cuadro 2 calculados con datos de los Censos Industriales de 1965 y 1970 a nivel de dos dígitos. De igual modo que con el coeficiente de Gini, cuando el índice de Herfindahal se aproxima a la unidad, el grado de desigualdad aumenta. En el cuadro 2 se puede observar que el grado de concentración varía de manera considerable entre industrias y que el nivel de concentración no ha cambiado de manera notable entre estos dos años considerados.

En la sección II, inciso 1 de este trabajo se analizan las causas que determinan el "tamaño mínimo óptimo de la empresa", concepto cuya vinculación con la concentración industrial se explica más adelante. Después se prueban algunas de las hipótesis postuladas en la literatura respecto a las causas de la concentración industrial. En particular se hacen estimaciones empíricas en relación a la importancia en términos absolutos y relativos, del tamaño óptimo de la empresa (sección II-2); sobre los requerimientos absolutos y relativos de capital por parte de las empresas (sección II-3); y sobre el tamaño de la industria y la tasa de crecimiento (sección II-4).

En la sección III se estudia la naturaleza de las relaciones entre concentración industrial, productividad de los factores y la intensidad en el uso de capital en la producción. Por último, en la sección IV se analizan los efectos de algunos de los factores que determinan el tamaño de las empresas en términos del número de empleados.

\section{Determinantes DEL GRAdo DE CONCENTRACión INDUSTRIAL}

El término "tamaño mínimo óptimo de la empresa" con frecuencia se utiliza para explicar el grado de concentración industrial. En la literatura sobre concentración industrial (Stigler, 1958; Savings, 1961; Weiss, 1964; Comanon y Wilson, 1967 y Scherer, 1973), el mínimo óptimo o tamaño más eficiente de la industria es aquel que corresponde al costo medio de producción mínimo. La mayor parte de los estudios empíricos en los cuales se estima un tamaño mínimo óptimo de la empresa se supone que la curva de costo medio de producción de largo plazo tiene 
forma de $L$. El tamaño mínimo óptimo se define entonces como aquel tamaño después del cual la curva de costos medios de largo plazo es horizontal. Las empresas de tamaño menor que el mínimo óptimo registran economías de escala crecientes a medida que aumentan de tamaño, y las empresas mayores al mínimo muestran economías de escala constantes.

El tamaño óptimo de la empresa puede ser un importante factor que determina la concentración industrial dado que en muchos casos sólo es posible alcanzar una escala eficiente de producción a tamaños tan grandes de empresas que hacen inevitable la concentración (Bain, 1959). Aún más, el tamaño mínimo óptimo a menudo es de tal magnitud que constituye una barrera a la entrada de nuevas empresas, lo cual contribuye a aumentar el grado de concentración.

En la primera parte de este apartado se analizan los factores que determinan el tamaño mínimo óptimo de las empresas en una industria. En la siguiente se estudian los modos en que el tamaño mínimo óptimo de la empresa y sus requerimientos de capital determinan el grado de concentración. Las relaciones entre una medida relativa del tamaño mínimo de la empresa y el grado de concentración industrial se consideran en el inciso siguiente $y$, por último, se estudian los efectos sobre el grado de concentración del tamaño absoluto de la industria así como su tasa de crecimiento y el grado de intensidad en el uso del capital de la industria.

\section{Factores que determinan el tamaño óptimo de la empresa}

Entre los factores que determinan el tamaño óptimo de la empresa, en los términos señalados antes, se pueden mencionar: el tamaño de la industria; la tasa de crecimiento de la industria; y la intensidad en el uso de capital en el proceso productivo.

a) El tamaño de la industria es un factor que determina el tamaño mínimo óptimo de la empresa dado que en una industria de gran magnitud, una empresa puede tener las ventajas de todas las economías de la producción disponibles. De acuerdo con este argumento, el tamaño mínimo óptimo de la empresa será mayor entre más grande sea el tamaño de la industria. El tamaño variable de la industria de empresa en términos del valor total de la producción para cada industria en un año dado.

b) Se puede argumentar que el tamaño mínimo óptimo de la empresa tiende a ser de gran magnitud en industrias que registran tasas rápidas de crecimiento. Esto debido a que es relativamente más fácil establecer una empresa grande en una industria en expansión que en otra estancada. La variable crecimiento se mide en función de la tasa de crecimiento de la producción de cada industria entre 1965 y 1970. 
c) En México, las empresas grandes por lo general utilizan métodos de producción intensivos en el uso de capital debido al conjunto de precios, relativamente distorsionado, al que se enfrentan (propiciado por las políticas proteccionistas) que estimulan el uso de técnicas intensivas en el uso de capital. Se puede argumentar que debido a esto, el tamaño mínimo óptimo de la empresa sería grande en industrias sumamente intensivas en el uso de capital más que en el uso de mano de obra. En este trabajo se mide el grado de intensidad en el uso del capital para cada industria mediante dos variables: la relación capital/trabajo y el valor de los combustibles y lubricantes por trabajador.

En esta sección, se hace una prueba mediante un análisis de regresión simple, sobre el grado de importancia que tienen las variables a), b) y c) mencionadas antes, en la determinación del tamaño mínimo óptimo de la empresa en una industria.

El tamaño mínimo óptimo de la empresa se estima mediante la técnica de "sobrevivencia" utilizada por Stigler (1958), Weiss (1964) y Savings (1961) en estudios sobre Estados Unidos y por Gollás (1978) para México. De acuerdo con esta técnica, el tamaño mínimo óptimo es la empresa media de la clase que ha ganado la mayor parte del mercado en la industria durante un cierto periodo de tiempo. Se estimó el tamaño mínimo óptimo de acuerdo a esta técnica con datos de los Censos Industriales de 1965 y de 1970 a nivel de cuatro dígitos. ${ }^{1}$

La especificación de la ecuación de regresión que ajustó mejor los datos es de la forma multiplicativa, la cual es lineal logarítmica. Esta especificación tiene la ventaja de que los coeficientes representan elasticidades con un claro sentido económico. La regresión estimada es:

$\ln M_{1}^{i}=2.35+0.50 \ln S_{i}+1.63 \ln \Delta Q_{i}+0.84 \ln \left(\frac{K}{L}\right)_{i}-0.33 \ln \left(\frac{F}{L}\right)_{i}$

$R^{2}=0.31 \quad F=5.15 \quad$ número de industrias $=50$

en donde:

$i=$ industrias;

$M_{1}=$ tamaño mínimo óptimo de la empresa estimado por la técnica de sobrevivencia;

$S=$ valor del producto (tamaño de la industria);

$\triangle Q=$ tasa de crecimiento del producto entre 1965 y 1970;

$(K / L)=$ relación capital/trabajo;

$(F / L)=$ relación valor de los combustibles y lubricantes/trabajo.

1 Para una explicación más detallada sobre el uso de este método y su aplicación al caso de México, véase Gollás (1978). 
Los coeficientes de regresión estimados resultaron con el signo esperado, y son estadísticamente significativos con excepción del coeficiente del valor de combustibles y lubricantes por trabajador. Las variables independientes explican alrededor del $30 \%$ de la variancia del tamaño mínimo óptimo de la empresa.

Dado que los coeficientes de la regresión utilizada representan elasticidades, éstas miden el grado de respuesta del tamaño mínimo óptimo a los cambios en las variables independientes. Por ejemplo, una industria que crece a una tasa $10 \%$ más rápido que otra tendrá un tamaño mínimo óptimo de empresa $16 \%$ mayor; o, una industria que tiene una relación capital/trabajo $10 \%$ mayor que otra, tendrá un tamaño mínimo óptimo de empresa $8.4 \%$ mayor. Se puede hacer una interpretación similar de las variables que miden el tamaño de la industria.

Los resultados de este trabajo apoyan la afirmación de que el tamaño mínimo óptimo de la empresa para una industria será mayor a medida que aumenta el tamaño de ésta, su tasa de crecimiento o su intensidad en el uso de capital.

Una vez analizados los factores que determinan el tamaño mínimo óptimo de la empresa, se estudiará de qué manera el tamaño de la empresa, y sus requerimientos de capital, afectan la concentración industrial.

\section{Tamaño minimo óptimo de la empresa y sus requerimientos absolutos} de capital como determinantes del grado de concentración industrial

Con frecuencia se explica el grado de concentración del producto o del empleo en una industria en función del tamaño mínimo óptimo de la empresa y de sus requerimientos absolutos de capital. El tamaño mínimo óptimo de la empresa es un factor que determina el grado de concentración industrial dado que constituye una barrera importante de entrada a la industria. A partir de estas consideraciones, se postula que el grado de concentración y el tamaño mínimo óptimo de la empresa están relacionados positivamente: entre más grande sea el tamaño mínimo óptimo de la empresa mayor será el grado de concentración y viceversa.

Los requerimientos absolutos de capital de la empresa de tamaño mínimo óptimo es otro factor que puede también ser un determinante del grado de concentración industrial (Comanor y Wilson, 1967; y Guth, 1971). Los requerimientos absolutos de capital agrandan la barrera de entrada de nuevas empresas, impiden la competencia e incrementan la concentración. Por esta razón, a menudo se afirma que el grado de concentración del producto y del empleo y los requerimientos absolutos de capital del tamaño óptimo de la empresa están positivamente relacionados.

En esta sección se prueba la hipótesis de que existe una relación positiva entre el grado de concentración industrial y: a) el tamaño mínimo 
óptimo de la empresa; y $b$ ) los requerimientos de capital de la empresa de tamaño mínimo óptimo.

La concentración en una industria se estima mediante tres índices: el coeficiente de Gini en términos del empleo $\left(C_{1}\right)$; el número de empresas de mayor tamaño que en conjunto producen el $80 \%$ del producto industrial $\left(C_{2}\right)$; y el número de las empresas mayores que dan empleo al $80 \%$ de la fuerza de trabajo $\left(C_{3}\right)$. Los índices $C_{2}$ y $C_{3}$ miden la concentración de manera inversa: entre más reducido sea su valor, mayor será el grado de concentración y viceversa.

El tamaño mínimo óptimo de la empresa se obtiene calculando primero el tamaño medio de la empresa, en términos del valor del producto, para las empresas de mayor tamaño que registran el $50 \%$ del producto total de una industria dada. Este tamaño medio de la empresa se divide luego entre el producto total de la industria para obtener el tamaño mínimo óptimo de la empresa. Estimado de este modo, el tamaño mínimo óptimo se expresa como porciento del producto de la industria. La estimación de este tamaño óptimo se hace por un procedimiento similar al utilizado por Comanor y Wilson (1967) y Guth (1971).

La variable requerimientos absolutos de capital se obtiene al multiplicar el tamaño medio de las empresas que producen el $50 \%$ del producto total de la industria por el cociente que resulta de dividir la inversión total de la industria (activos) entre el producto total. Los requerimientos absolutos de capital calculados se expresan en miles de pesos.

Los resultados de las ecuaciones de regresión lineales logarítmicas, se muestran en el cuadro 3. Todos los coeficientes tienen el signo esperado y son estadísticamente significativos. En todas las regresiones aproximadamente $50 \%$ de la varianza de la concentración es explicada por el tamaño mínimo óptimo de la empresa y por sus requerimientos absolutos de capital. En las regresiones (2) y (3), como se esperaba, los coeficientes resultaron con signo negativo, dado que los índices de concentración $C_{2}$ y $C_{3}$ miden, en sentido inverso, el grado de concentración, de acuerdo con la regresión (2). Dado que los coeficientes representan elasticidades, los resultados sugieren que, por ejemplo, un aumento del $10 \%$ en el tamaño mínimo óptimo de la empresa incrementaría la concentración en $8.5 \%$; y que un incremento del $10 \%$ en los requerimientos absolutos de capital, incrementarían la concentración en $1.8 \%$. Con los resultados de las otras regresiones se pueden hacer interpretaciones semejantes.

Estos resultados empíricos apoyan el punto de vista de que un incremento en el tamaño mínimo óptimo de la empresa o en los requerimientos absolutos de capital de la empresa de tamaño mínimo óptimo, incrementará el grado de concentración y viceversa. 
Cuadro 3

ECUACIONES DE REGRESIÓN MÚLTIPLE UTILIZADAS PARA MEDIR LA CONCENTRACIÓN,a' 1970

\begin{tabular}{|c|c|c|c|c|}
\hline \multirow{2}{*}{\multicolumn{2}{|c|}{$\begin{array}{c}\text { Variables independientes } \\
y \text { otros indices }\end{array}$}} & \multicolumn{3}{|c|}{ Regresiones } \\
\hline & & $\begin{array}{c}c_{1} \\
(1)\end{array}$ & $\begin{array}{c}c_{2} \\
(2)\end{array}$ & $\begin{array}{c}c_{3} \\
(3)\end{array}$ \\
\hline & $\mathrm{M}_{2}$ & $\begin{array}{l}0.127 \\
(0.021) \mathrm{b} /\end{array}$ & $\begin{array}{c}-0.85 \\
(0.076) \mathrm{b}\end{array}$ & $\begin{array}{l}-0.80 \\
(0.084) \mathrm{b}\end{array}$ \\
\hline & $\mathrm{KR}$ & $(0.73)$ & $\begin{array}{l}-0.18 \\
(0.043)^{\mathrm{b}} \prime\end{array}$ & $\begin{array}{c}-0.20 \\
(0.048) \mathrm{b}\end{array}$ \\
\hline & $R^{2}$ & 0.43 & 0.57 & 0.51 \\
\hline & $\mathbf{r}$ & 74 & 131 & 105 \\
\hline Nimero de & industrias & 201 & 201 & 201 \\
\hline
\end{tabular}

a El índice de concentración $C_{1}$ expresa el coeficiente de Gini en términos del empleo; $C_{2}$ el número de empresas que genera el $80 \%$ de la producción industrial y; $C_{3}$ el número de empresas que da empleo al $80 \%$ de la fuerza de trabajo industrial. Para la definición y unidades de medida utilizadas para calcular el tamaño mínimo óptimo de la empresa $\left(M_{2}\right)$ y sus requerimientos de capital $(K R)$, véase el texto.

b Errores estándar.

\section{Tamaño minimo óptimo relativo de la empresa y grado} de concentración industrial

La naturaleza de la relación entre el grado de concentración y el tamaño óptimo puede analizarse además mediante el concepto de tamaño óptimo relativo de la empresa en contraposición al tamaño absoluto. Conforme a esta idea, el cambio en el tamaño óptimo de la empresa en relación al de la industria, es la variable explicativa importante de cambio en la concentración. Esto es, el tamaño mínimo óptimo relativo de la empresa se obtiene al estimar un tamaño mínimo óptimo y ponderar esta cifra por el tamaño de la industria. Este cálculo se hace como sigue: al contrario del tamaño de empresa utilizado por Weiss (1963), el tamaño mínimo óptimo implica la estimación del tamaño que corresponde al "punto medio" de la empresa, o sea el punto medio de la distribución ordenada del producto. El "punto medio" del tamaño de la empresa se estima de la siguiente manera: la suma del valor agregado de todas las clases de tamaño respecto al empleo se calculó para encontrar la clase que contenía a la empresa de "tamaño medio". El tamaño de la empresa a la que correspondía el punto medio en función del empleo se estimó por interpolación. La empresa del punto medio ya en 
términos del valor agregado, se determinó al multiplicar su número de empleados por el valor agregado por trabajador en dicha clase de tamaño. Una vez obtenido el "punto medio" del tamaño de empresa se ponderó éste por el tamaño de la industria. El cambio del tamaño óptimo relativo de la empresa se calculó como sigue:

$$
\triangle M_{3}^{i}=\frac{T P M E(1970)}{T I(1970)} \div \frac{T P M E(1965)}{T I(1965)}
$$

en donde: $T P M E=$ tamaño que corresponde al "punto medio" de la empresa;

$T I=$ tamaño de la industria.

El tamaño de "punto medio" y el tamaño de la industria se miden en términos del valor agregado en cada industria $i$.

El cambio en el nivel de concentración entre 1965 y 1970 se estimó a través del cambio en el valor del coeficiente de Gini $\left(\Delta C_{1}^{i}\right)$ para cada industria $i$.

Se estimó una regresión lineal logarítmica en la que se relacionan la tasa de cambio de la concentración y el tamaño de la empresa que corresponde al punto medio con respecto al tamaño de la industria. Se obtuvieron los siguientes resultados:

$$
\begin{aligned}
& \ln C_{1}^{i}=1.03+\begin{array}{l}
0.312 \ln \Delta M_{3}^{i} \\
(0.051)
\end{array} \\
& R^{2}=0.17 \quad F=37.0 \quad \text { Número de industrias }=185
\end{aligned}
$$

Estos hallazgos apoyan el punto de vista de que el grado de concentración en una industria y el tamaño óptimo relativo están relacionados positivamente: un incremento en el tamaño mínimo óptimo relativo incrementa el nivel de concentración y viceversa.

\section{Tasa de crecimiento, intensidad en el uso de capital y tamaño}

de la industria como barreras de ingreso que originan la concentración industrial

Se pueden investigar las causas de la concentración del producto y del empleo en una industria a través del análisis de las relaciones entre concentración y: a) el tamaño absoluto de la industria; b) su tasa de crecimiento; y c) su grado de intensidad en el uso de capital.

Con frecuencia se argumenta que cuando el tamaño de una industria es de gran magnitud, existen mayores oportunidades para la entrada de nuevas empresas y se reduce así la concentración. Se considera también que en industrias con un rápido crecimiento es probable que exista un 
grado reducido de concentración, dado que, a medida que se abren nuevos mercados, aumentan las oportunidades para la entrada de nuevas empresas a la industria. Sin embargo, también se puede esperar que ocurra lo contrario: entre mayor sea el tamaño de la industria y mayor sea su tasa de crecimiento, mayor será la probabilidad de que las em. presas grandes, bien establecidas, puedan crecer aún más y aumentar así la concentración. Dicho de otra manera, industrias de gran magnitud y con un crecimiento acelerado facilitan el aprovechamiento de las ventajas de las economías de escala por parte de las empresas grandes y en consecuencia crecerán aún más y aumentará el grado de concentración.

El grado de intensidad en el uso de capital en una industria es uno de los factores que determinan su grado de concentración. Se puede argumentar que en una industria intensiva en alto grado en el uso de capital, existe una sustancial indivisibilidad en el equipo de capital que estimula el crecimiento de empresas de gran tamaño y contribuye así a aumentar la concentración. Esta tendencia se refuerza adicionalmente si se considera que en México las empresas nuevas y de tamaño reducido, con frecuencia no disponen de facilidades de crédito, derechos preferenciales de importación, etc. de que disfrutan las grandes empresas. Bajo estas circunstancias, sólo se estimula la expansión de empresas grandes y bien establecidas y, en consecuencia se incrementa la concentración.

En esta sección se prueba empíricamente: a) la dirección e intensidad del efecto que tiene el tamaño de la industria y su tasa de crecimiento sobre la concentración industrial; y b) la hipótesis de que la intensidad en el uso de capital en la producción y la concentración están relacionados inversamente.

Las variables utilizadas para medir el tamaño de las industrias son los valores de sus activos o su número total de trabajadores. El aumento en el tamaño de las industrias se estimó en términos de la tasa de crecimiento de su producto entre 1965 y 1970 . La variable que expresa el grado de intensidad de uso de capital en cada industria es el valor de la relación capital trabajo. La concentración del producto $\left(C_{2}\right)$ se estimó con el número de empresas de mayor tamaño que en conjunto producían $80 \%$ del producto de la industria en 1970. La concentración del empleo $\left(C_{3}\right)$ se midió con el número de empresas de mayor tamaño que en conjunto dieron empleo al $80 \%$ de la fuerza de trabajo en cada industria en dicho año. Tanto $C_{2}$ como $C_{3}$ miden la concentración en forma inversa: entre más grande sea su valor, mayor será el grado de concentración y viceversa.

La ecuación de regresión lineal logarítmica, estimada por mínimos cuadrados fue la siguiente:

$$
\ln C_{2}^{i}=1.72+0.56 \ln A_{i}-1.28 \ln \left(\frac{K}{L}\right)_{i}-0.73 \ln \triangle Q_{i}
$$




$$
\begin{aligned}
& R^{2}=0.47 \quad F=62 \quad \text { Número de industrias }=183 \\
& \ln C_{3}^{i}=2.46+0.50 \ln L_{i}-0.74 \ln \left(\frac{K}{L}\right)_{i}-0.039 \ln \triangle Q_{i} \\
& \begin{array}{lll}
(0.069) & (0.098) & (0.156)
\end{array} \\
& R^{2}=0.37 \quad F=33 \quad \text { Número de industrias }=176
\end{aligned}
$$

En donde:

$i=$ industrias;

$C=$ índice de concentración del producto o del empleo;

$A=$ valor de los activos totales de la industria (tamaño de la industria);

$L=$ tamaño de la fuerza de trabajo (tamaño de la industria);

$K / L=$ relación capital/trabajo;

$\Delta Q=$ tasa de crecimiento del producto.

Los resultados de la ecuación (1) indican que el $47 \%$ de la variación en la concentración del producto es "explicada" por cambios en las variables independientes; mientras que los de la regresión (2) indican que la variación explicada de la concentración del empleo es de $37 \%$.

Los signos de los coeficientes que miden el tamaño absoluto de las industrias (valor total de los activos o número total de trabajadores), es positivo y estadísticamente significativo en ambas regresiones. Estos resultados apoyan el punto de vista de que es posible encontrar niveles bajos de concentración del producto o del empleo en las industrias de gran magnitud. Por ejemplo, los resultados de la regresión (1) sugieren que una industria que fue $10 \%$ mayor que otra (en términos del valor total de sus activos), estaría $5.6 \%$ menos concentrada y los resultados de la ecuación (2) sugieren que una industria que emplea $10 \%$ más de trabajadores que otra estará $5 \%$ menos concentrada en términos del empleo.

El signo negativo de los coeficientes que miden el grado de intensidad en el uso de capital es significativo en ambas regresiones. Esto sugiere que en industrias con un alto grado de intensidad en el uso de capital es probable que exista un alto grado de concentración del producto y del empleo. Sin embargo, el valor de la elasticidad de la concentración del producto con respecto a la relación capital trabajo es mayor que el de la concentración del empleo respecto a la relación capital/trabajo. Así, un incremento del 10\% en el grado de intensidad en el uso de capital, probablemente incremente alrededor de $12.8 \%$ la concentración del producto y un $7.4 \%$ en la concentración del empleo. En otras palabras, un incremento en la intensidad de uso de capital en una industria incrementaría proporcionalmente más la concentración del producto que la del empleo. 
Dado que los coeficientes de la tasa de crecimiento de las industrias no son estadísticamente significativos en ambas regresiones, no se puede decir mucho acerca de la relación entre concentración y tasa de crecimiento de las industrias. Sin embargo, se intentará analizar más adelante esta relación.

Con frecuencia se argumenta que un crecimiento rápido alienta la entrada de nuevas empresas a la industria ante la perspectiva de obtener ganancias elevadas y porque las barreras a la entrada pueden parecer menos difíciles de superar en una industria en expansión. Por estas razones se considera que el crecimiento de la industria y su nivel de concentración están relacionados de manera inversa. Algunos estudios empíricos (Nelson, 1960; Sheperd, 1964; y Sawyer, 1971) apoyan este punto de vista, aunque alguna evidencia contraria (Kamerschen, 1968) lo refuta.

Para probar la hipótesis de que existe una relación inversa entre la tasa de crecimiento de una industria y su nivel de concentración se utiliza la tasa de cambio del producto de la industria como variable de crecimiento y la tasa de crecimiento del número de empresas en la industria como la variable que expresa la barrera de entrada. Si las barreras de entrada son fáciles de superar, se puede esperar un crecimiento rápido del número de empresas en la industria y viceversa. Las variables que miden los cambios en el nivel de concentración son las tasas de cambio de $C_{2}$ y $C_{3}$ cuyos valores, como se expresa antes, miden la concentración en sentido inverso.

Los resultados de las ecuaciones lineales y lineal logarítmicas estimados se muestran en el cuadro 4. Los coeficientes estimados resultaron con signo negativo para la tasa de crecimiento del producto y positivo para la tasa de crecimiento del número de empresas. Todos los coeficientes son estadísticamente significativos $\mathrm{y}$, en todas las regresiones, los cambios en las variables independientes explican por lo menos un $35 \%$ de la variación en la tasa de crecimiento de la concentración.

Los resultados obtenidos constituyen evidencia en apoyo del punto de vista de que las barreras de entrada a una industria explican en buena medida el grado de concentración. Por ejemplo, de acuerdo con la ecuación (3) (véase el cuadro 4), si la tasa de crecimiento del número de empresas en una industria dada es $10 \%$ mayor que en otra (es decir, las barreras a la entrada no son tan difíciles de salvar) se esperaría que la tasa de cambio en la concentración fuera $7.6 \%$ más baja. La incorporación a ritmo acelerado de nuevas empresas en una industria atenúa el aumento de la concentración.

Por otro lado, el signo y la magnitud del coeficiente de $\Delta Q$ sugiere que la concentración tiene mayores probabilidades de incrementarse en industrias donde se registra un ritmo de expansión más acelerado. Por ejemplo, conforme a la regresión (3) (véase el cuadro 4), una industria 
con un ritmo de crecimiento $10 \%$ más rápido que el de otra tendría una tasa de crecimiento de la concentración $1.9 \%$ mayor y, de acuerdo con la ecuación (2), una tasa de incremento en la concentración del empleo $1.3 \%$ mayor. De esta manera, nuestros resultados no respaldan el punto de vista de que las deseconomías de escala y otros obstáculos afectan de manera adversa la capacidad de crecimiento de las empresas

\section{Cuadro 4}

ECUACIONES DE REGRESIÓN MÚLTIPLE QUE RELACIONAN CAMBIOS EN EL NIVEL DE CONCENTRACIÓN, CRECIMIENTO DE LA PRODUCCIÓN Y TASA DE CRECIMIENTO DEL NÚMERO DE EMPRESAS EN UNA INDUSTRIA

\begin{tabular}{lccccc}
\hline $\begin{array}{c}\text { Variables } \\
\text { independientes }\end{array}$ & $\Delta Q$ & $\Delta N F$ & $R^{2}$ & $F$ & $\begin{array}{c}\text { Munero de } \\
\text { industrias }\end{array}$ \\
\hline (1) $\Delta C_{2}$ & $\begin{array}{c}-0.080 \\
(0.029)\end{array}$ & $\begin{array}{c}0.75 \\
(0.070)\end{array}$ & 0.38 & 56 & 190 \\
(2) $\Delta C_{3}$ & $\begin{array}{c}0.091 \\
(0.030)\end{array}$ & $\begin{array}{c}0.98 \\
(0.073)\end{array}$ & 0.49 & 91 & 192 \\
\hline
\end{tabular}

\begin{tabular}{lccccc}
\hline & ln $Q$ & ln NF & $R^{2}$ & $E$ & $\begin{array}{c}\text { MGmero de } \\
\text { industrias }\end{array}$ \\
\hline (3) In $\Delta c_{2}$ & $\begin{array}{c}-0.13 \\
(0.070)\end{array}$ & $\begin{array}{c}0.76 \\
(0.081)\end{array}$ & 0.35 & 49 & 190 \\
(4) In $\Delta c_{3}$ & $\begin{array}{c}-0.19 \\
(0.062)\end{array}$ & $\begin{array}{c}0.94 \\
(0.073)\end{array}$ & 0.49 & 91 & 192 \\
\hline
\end{tabular}

a $\triangle C_{2}$ y $\triangle C_{3}$ son las tasas de cambio de los índices de concentración de la producción y del empleo conforme se definen en el texto. $\triangle Q$ y $\triangle N F$ se refieren a las tasas de cambio de la producción y del número de empresas en la industria entre 1965 y 1970 . La parte superior del cuadro muestra los resultados de las regresiones lineales y la parte inferior los de las regresiones logarítmicas lineales.

grandes (Penrose, 1959; Baumol, 1962) o que "el crecimiento de los mercados industriales hace posible obtener mayores ventajas de la división del trabajo tanto en términos de industria como funcionales, y conduce a bajos niveles de concentración" (Nelson, 1960, p. 641). La asociación directa encontrada en el sector industrial de México entre crecimiento y concentración puede ser explicada si se considera que las empresas grandes en industrias con un ritmo acelerado de expansión están en mejor situación que las pequeñas para obtener poder monopólico e incrementar así el grado de concentración.

A continuación se hace un análisis de la naturaleza de la interrela- 
ción entre el aumento en el número de empresas de nueva incorporación y la tasa de crecimiento de la industria. También se estudian las interrelaciones entre concentración y aumento en el número de nuevas entradas cuando éste se considera como única variable explicativa en la ecuación de regresión (compárese esta formulación con la regresión del cuadro 4).

A continuación se incluye la regresión estimada entre las tasas de crecimiento del producto $(\triangle Q)$ y del número de nuevas empresas $(\triangle N F)$ y la regresión entre las tasas de crecimiento de nuevas empresas y la del grado de concentración $\left(\triangle C_{2}\right)$ :

$$
\begin{aligned}
& \text { ln } \triangle N F=0.45+0.37 \ln \Delta Q \\
& \text { (0.035) } \\
& R^{2}=0.36 \quad F=109 \quad \text { número de industrias }=200 \\
& \Delta C_{2}=0.29+0.71 \Delta N F \\
& (0.067) \\
& R^{2}=0.38 \quad F=113 \quad \text { número de industrias }=185 \\
& \text { ln } \triangle Q=1.26+1.98 \ln \triangle N F \\
& \text { (0.056) } \\
& R^{2}=0.86 \quad F=237 \quad \text { número de industrias }=200
\end{aligned}
$$

Los coeficientes obtenidos de la regresión (1) sugieren la existencia de una relación positiva entre incrementos en el número de nuevas entradas y la tasa de crecimiento del producto de la industria. Estas estimaciones indican también (regresión 2) que existe una relación negativa entre aumentos en el número de nuevas empresas y concentración en la industria (recuérdese que $C_{2}$ mide la concentración en sentido inverso).

De estos resultados se puede inferir que un incremento en la tasa de crecimiento del producto de una industria reducirá la concentración a través del efecto que dicho incremento tiene sobre el crecimiento del número de empresas. A primera vista, este resultado parece inconsistente con los resultados mostrados en el cuadro 4 , los cuales indican que una tasa de crecimiento rápido del producto aumenta el grado de concentración. Estos resultados contradictorios en apariencia, pueden ser explicados y aun reconciliados si se tienen en cuenta las consideraciones siguientes:

Aun cuando los aumentos en la tasa de crecimiento del producto industrial de México han estimulado la entrada de nuevas empresas y se hạ reducido así la concentración, al parecer también sucedió que la mayor parte del aumento en el producto es atribuible a las empresas más grandes, y esto ha agudizado la concentración. Esto resulta porque las ecuaciones en donde se incluyeron las tasas de crecimiento del pro- 
ducto de la industria y del número de nuevas empresas (véase el cuadro 4), muestran que un incremento en la tasa de crecimiento del producto eleva el grado de concentración mientras en forma simultánea alienta la entrada de nuevas empresas y contribuye así a reducir la concentración.

\section{CONCENTRACIÓN Y PRODUCTIVIDAd DE LOS FACTORES}

A menudo se afirma que en la mayor parte de los países subdesarrollados hay escasez de capital y abundancia de mano de obra. Dada esta dotación de factores, se logra un alto nivel de eficiencia cuando la productividad del capital es elevada. Desde el punto de vista de las políticas para influir sobre la concentración, lo importante es determinar si las empresas pequeñas utilizan menos capital y menores cantidades de otros factores escasos que las empresas grandes para producir un nivel dado de producto. La mayor parte de la evidencia disponible sugiere que las empresas pequeñas tienden a utilizar menos capital por unidad de producto que las grandes (Ranis, 1962; Marsden, 1969; Meheta, 1969; Todd, 1971; y Berry, 1972). Sin embargo, alguna evidencia en contra muestra que tanto la productividad del trabajo como la del capital aumentan con el tamaño de la empresa (Dhar y Lydall, 1961; Boon, 1964; Sanderasa, 1966 y 1969; y Cardwel, 1978). Además, dado que en general la productividad del trabajo (relación producto/trabajo) tiende a ser más elevada en las empresas grandes que en las de tamaño pequeño o medio; y dado que una industria con pocas empresas grandes por lo común registra un alto grado de concentración, se concluye que la productividad del trabajo se incrementa con la concentración. Si se acepta el argumento de que las empresas pequeñas son más eficientes en el uso intensivo del escaso capital (bajas relaciones capital/producto) y también de que hacen uso extensivo de la abundante mano de obra (bajas relaciones capital/trabajo), entonces conviene reducir el nivel de concentración, o sea aumentar el número de empresas pequeñas y de tamaño medio en la economía.

El punto de vista de que las empresas pequeñas y medianas utilizan técnicas más intensivas en el uso de mano de obra (baja relación capital/ trabajo) que las de tamaño grande es apoyada por la mayor parte de los estudios empíricos (Dhar y Lidall, 1961; Ranis, 1961; Shetty, 1963; Marsden, 1966; Berry, 1972; Ditullio, 1972). Las razones aducidas de manera usual para explicar el uso de técnicas intensivas en el uso de capital por las empresas de tamaño pequeño y mediano son que las empresas de este tipo enfrentan condiciones más competitivas que las empresas de gran tamaño y se ven forzadas a elegir una tecnología más acorde con el factor abundante en la economía. En otras palabras, las 
empresas pequeñas se enfrentan con un conjunto de precios relativos de los factores más cercanos a los precios reales de escasez que los que afrontan las empresas grandes. Por otro lado, las empresas grandes tienden a ser menos intensivas en el uso de mano de obra y a pagar salarios elevados debido a la presencia de sindicatos poderosos y porque la legislación sobre salarios es más efectiva en las empresas grandes que en las pequeñas. Garbelino (1950) Weiss (1966) y Philips (1971) han encontrado que las grandes empresas en industrias con más alto grado de concentración pagan salarios más elevados que las empresas en industrias de menor concentración. Otro factor que explica el grado de intensidad en el uso de capital en las empresas grandes es que el crédito subsidiado y otros subsidios al capital, tales como el tratamiento fiscal preferencial así como facilidades para importar capital están al alcance para la mayoría de éstas.

De acuerdo con el argumento anterior, los salarios tienden a ser bajos (altos) y los costos del capital elevados (bajos) para empresas de tamaño pequeño (grande) y por lo tanto tienden a adoptar técnicas intensivas en el uso del trabajo (capital). Si es cierto que en las empresas pequeñas predominan técnicas intensivas en el uso de mano de obra, no porque operen a pequeña escala sino porque enfrentan un conjunto menos distorsionado de precios relativos de los factores que las empresas grandes, se puede argüir que las políticas para incrementar el empleo no deben propiciar la creación de más empresas de tamaño pequeño (reducir la concentración) sino que deben procurar la eliminación las distorsiones en los precios de los factores que dan lugar a mercados de factores duales. En caso de tener éxito estas políticas, las empresas de cualquier tamaño adoptarán técnicas intensivas en el uso de trabajo.

En esta sección se presenta una estimación preliminar de la interrelación entre el grado de concentración en el sector industrial de México y: a) el crecimiento del empleo; b) la productividad de la mano de obra; c) el nivel de salarios; y d) la intensidad en el uso de capital en la producción.

Los resultados de la regresión lineal logarítmica estimada, se muestran en el cuadro 5. El grado de concentración se estima, al igual que antes, mediante $C_{2}$ y $C_{3}$ (el número de empresas grandes que en conjunto generan el $80 \%$ del producto de la industria y el de las empresas más grandes que en conjunto dan empleo al $80 \%$ de la fuerza de trabajo, respectivamente; ambas miden el grado de concentración en sentido inverso). Dado que en México se ha constatado la existencia de una industria con un alto grado de concentración, es probable que también sea una industria con un número reducido de empresas grandes (Gollás, 1978) y por tanto en este trabajo nos referimos a la industria altamente concentrada como una en la que existen pocas empresas de gran tamaño. Las otras variables utilizadas en la regresión tienen la conno- 
tación usual: para cada industria, $Q$ representa el valor agregado, $L$ el número de trabajadores, $W$ se refiere a los salarios y $K$ al valor total de los activos.

Los resultados de la ecuación (1), señalan la existencia de una relación inversa entre el grado de concentración y el crecimiento del empleo en la industria. El hecho de que el coeficiente (elasticidad) sea estadísticamente significativo sugiere que es factible un incremento de un $2.8 \%$ la tasa de crecimiento del empleo si la tasa de crecimiento en el grado de concentración disminuye en $10 \%$. Además, el valor de $R^{2}$ indica que más del $50 \%$ de la variación en el ritmo de crecimiento del empleo se explica por el crecimiento del nivel de concentración industrial.

Con base en los resultados de las ecuaciones (2), (3) y (4), se infiere que a medida que aumenta el grado de concentración, la productividad del trabajo. los salarios industriales y la intensidad en el uso de capital también aumentarán. Los coeficientes estimados sugieren, por ejemplo, que si una industria tiene un grado de concentración $10 \%$ mayor que otra podría: a) pagar salarios $1.8 \%$ más elevados; b) utilizar una tecnología $3.5 \%$ más intensiva en el uso de capital; y c) operar con una fuerza de trabajo $2.3 \%$ más productiva.

El efecto reductor que sobre la tasa de empleo tiene un incremento en la tasa de concentración (ecuación 1), probablemente se debe a que el mismo incremento de la concentración también eleva el nivel de salarios (ecuación 3) con lo cual se reduce así la tasa de empleo. En otras palabras, el aumento en el precio del trabajo, originado por un incremento en el grado de concentración, reduce la tasa de crecimiento del empleo.

Sin embargo, la dirección de la relación de causalidad entre concentración y grado de intensidad en el uso de capital puede tener sentido inverso: se puede argumentar que la adopción de técnicas altamente intensivas en el uso de capital estimulan la concentración, y no que un incremento del grado de concentración induce la adopción de técnicas altamente intensivas en el uso de capital, como sugieren los resultados de la ecuación 4 (véase el cuadro 5). Las regresiones (5), (6) y (7) indican que si el nivel o la tasa de cambio en la intensidad de uso de capital en la producción aumentan, es probable que se de un grado de concentración elevado.

En resumen, puede decirse que estos resultados apoyan el punto de vista de que las industrias constituidas por empresas pequeñas (reducido nivel de concentración), al contrario de lo que sucede en las industrias con predominio de empresas grandes tienden: 1) a registrar mayores tasas de crecimiento del empleo y menor productividad de la mano de obra; 2) a pagar salarios bajos; y 3) a usar técnicas de producción intensivas en mano de obra. 


\section{Cuadro 5}

REGRESIONES EN DONDE SE RELACIONAN LAS MEDIDAS DE LA PRODUCTIVIDAD DE LOS FACTORES, LA INTENSIDAD EN EL USO DE CAPITAL Y LA CONCENTRACIÓN

\begin{tabular}{|c|c|c|c|c|c|c|c|c|}
\hline \multicolumn{2}{|c|}{$\begin{array}{l}\text { Variables } \\
\text { independientes }\end{array}$} & \multirow[t]{2}{*}{$c_{2}$} & \multirow{2}{*}{$\frac{c_{2}}{(0.29}$} & \multirow[t]{2}{*}{$\frac{K}{2}$} & \multirow[t]{2}{*}{$\frac{K}{\mathbf{L}}$} & \multirow{2}{*}{$\frac{\mathrm{R}^{2}}{0.54}$} & \multirow{2}{*}{$\frac{F}{237}$} & \multirow{2}{*}{$\begin{array}{l}\begin{array}{l}\text { Namero de } \\
\text { industrias }\end{array} \\
96\end{array}$} \\
\hline (1) & L & & & & & & & \\
\hline (2) & $\frac{Q}{L}$ & $\begin{array}{l}-0.23 \\
(0.040)\end{array}$ & & & & 0.26 & 33 & 96 \\
\hline (3) & $w$ & $\begin{array}{l}-0.18 \\
(0.029)\end{array}$ & & & & 0.29 & 38 & 96 \\
\hline (4) & $\frac{K}{L}$ & $\begin{array}{l}-0.35 \\
(0.048)\end{array}$ & & & & 0.37 & 53 & 91 \\
\hline (5) & $c_{2}$ & & & $\begin{array}{l}-1.07 \\
(0.147)\end{array}$ & & 0.37 & 53 & 91 \\
\hline (6) & $c_{3}$ & & & $\begin{array}{l}-1.04 \\
(0.153)\end{array}$ & - & 0.34 & 46 & 91 \\
\hline (7) & $c_{2}$ & & & & $\begin{array}{l}-0.62 \\
(0.131)\end{array}$ & 0.19 & 22 & 96 \\
\hline (8) & $c_{3}$ & & & & $\begin{array}{l}-0.77 \\
(0.135)\end{array}$ & 0.26 & 32 & 96 \\
\hline
\end{tabular}

a Todas las regresiones son logaritmicas lineales. Los errores estándar aparecen entre paréntesis.

\section{Determinantes del tamaño de la empresa en Función DEL EMPLEO}

Los factores que determinan el tamaño de la empresa en términos del número de empleados se puede estudiar a través del concepto de tamaño mínimo óptimo de la empresa estimado mediante la técnica de "sobrevivencia"; como se explicó antes, la determinación del tamaño mínimo óptimo de la empresa para cada industria mediante la técnica de sobrevivencia consiste en encontrar el tamaño medio de la empresa para la clase que ha logrado el control mayoritario del mercado en un cierto periodo de tiempo.

Se puede aplicar la misma técnica para determinar el tamaño óptimo de la empresa, ahora en términos del empleo, para la clase que ha registrado el mayor incremento en el empleo durante cierto periodo de tiempo.

Una vez que se determina el tamaño de la empresa cuyo crecimiento del empleo ha sido el más rápido, se puede preguntar acerca de los factores que contribuyen a determinar su tamaño. Entre estos factores se puede mencionar el mercado de trabajo en la industria; la tasa de cre- 
cimiento del producto de la industria; y la intensidad en el uso de capital en la industria. Los mecanismos a través de los cuales estos factores afectan el tamaño de la empresa en términos del empleo son:

a) Si una empresa opera en una indusria que emplea a un gran número de trabajadores, es muy probable que en el mercado particular de trabajo los precios de éste sean lo suficiente reducidos como para que hagan atractivo utilizar técnicas intensivas en el uso de capital. En este trabajo se prueba la hipótesis de que el tamaño de la empresa en términos del número de empleados será de gran magnitud si es también grande el mercado de trabajo en el cual operan las empresas.

b) Un crecimiento rápido de la industria posibilita que las empresas aprovechen las ventajas de las economías de escala en la producción y se estimule el crecimiento de las empresas. En este estudio se somete a prueba la hipótesis de que existe una relación positiva entre el tamaño de la empresa (según el número de trabajadores) con la tasa más elevada de crecimiento del empleo y la tasa de crecimiento de la industria correspondiente.

c) El efecto que tiene el grado de intensidad en el uso de capital en la producción sobre el monto del empleo de las empresas que han experimentado la mayor tasa de crecimiento del empleo es más difícil de determinar. En algunos casos, se encuentran empresas de gran tamaño en términos del empleo en industrias en las que la intensidad en el uso de mano de obra es baja, aunque con frecuencia también se encuentran en industrias intensivas en el uso de capital. En este trabajo se prueba si es más probable encontrar empresas de gran tamaño, en términos del empleo, en industrias con alta o baja intensidad en el uso de capital.

El tamaño del mercado de trabajo en cada industria se mide mediante el número total de trabajadores en 1965 y 1970 . La tasa de crecimiento de cada industria se mide con la tasa de crecimiento del producto. La intensidad de uso de capital se mide a través de dos variables: la relación capital/trabajo y el valor de los combustibles y lubricantes utilizados por trabajador ocupado.

Los resultados obtenidos del ajuste de los datos, mediante una regresión logarítmica lineal, fueron los siguientes:

$$
\begin{aligned}
\ln (F S)_{i}= & 2.8+0.52 \ln L_{i}+0.83 \ln \Delta Q_{i}+0.47 \ln \left(\frac{K}{L}\right)- \\
& (0.15) \quad(0.47) \\
& -0.17 \ln \left(\frac{F}{L}\right)
\end{aligned}
$$

$$
R^{2}=0.25 \quad F=4.1 \quad \text { Número de industrias }=55
$$


en donde:

$$
\begin{aligned}
i= & \text { industrias; } \\
F S= & \text { tamaño medio de la empresa de la clase que proporcional- } \\
& \text { mente generó más empleo entre } 1965 \text { y } 1970 ; \\
L= & \text { tamaño del mercado de trabajo; } \\
\triangle Q & =\text { tasa de crecimiento del producto entre } 1965 \text { y } 1970 \\
K / L= & \text { relación capital/trabajo; } \\
F / L= & \text { valor de los combustibles y lubricantes por trabajador. }
\end{aligned}
$$

Los coeficientes de las variables que miden el tamaño del mercado de trabajo y la tasa de crecimiento de la industria resultaron con el signo esperado y son estadísticamente significativas. El coeficiente que corresponde a lubricantes y combustibles no es estadísticamente significativo. Estos resultados sugieren que es probable encontrar que la empresa que genera proporcionalmente más empleo es más grande, en términos del número de trabajadores, en industrias con un mercado de trabajo relativamente grande y con una tasa acelerada de crecimiento del producto. Por ejemplo, el tamaño en términos del número de trabajadores de la empresa que en proporción genera más empleo es probable que sea $8.3 \%$ mayor en una industria que crece $10 \%$ más rápido que otra.

El signo positivo del coeficiente de la relación capital/trabajo, sugiere que el tamaño, en términos del número de trabajadores, de la empresa que proporcionalmente genera más empleo, probablemente sea mayor en industrias más intensivas en el uso de capital. Este hecho es razonable si se considera que en México muchas de las políticas económicas (facilidades a la importación de capital, subsidios al capital, facilidades de crédito y exenciones impositivas, entre otras) tradicionalmente han alentado el crecimiento rápido de empresas grandes que emplean técnicas intensivas en el uso de capital. Estos estímulos al uso de técnicas intensivas en el uso de capital se han seguido de manera independiente de los requerimientos tecnológicos de la producción y de las disponibilidades relativas de factores productivos en el país. Las políticas proteccionistas que han propiciado el crecimiento rápido de empresas grandes han tenido un efecto dual sobre el empleo en la industria. Por un lado, los aumentos observados en la relación capital/trabajo de las grandes empresas industriales en México implican, por definición, que las empresas grandes utilizan menos trabajo por unidad de capital; sin embargo, debido a las políticas proteccionistas mencionadas antes, simultáneamente su producto ha crecido lo bastante rápido como para compensar el efecto depresivo de la capitalización sobre el empleo. No es extraño entonces encontrar que el tamaño de las empresas, en términos del número de trabajadores, que proporcionalmente generan más empleos es relativamente mayor en las industrias más intensivas en el uso de capital. 
Los resultados de este estudio sugieren que debe darse mayor importancia a la capacidad de generación de empleo de las empresas grandes que utilizan técnicas más intensivas en el uso de capital. No es del todo obvio que la creación de un gran número de empresas pequeñas intensivas en el uso de mano de obra es el único o el mejor camino para incrementar el empleo total.

\section{BIBLIOGRAFIA}

Bain, J. S. (1959), Industrial Organization, Nueva York, Wiley.

Baumol, W. J. (1962), "On the Theory of the Expansion of the Firm", American Economic Review, Vol. 52.

Berry, R. A. (1972), "The Relevance and Prospects of Small Scale Industry In Colombia", Economic Growth Center, Discussion Paper No 142, septiembre.

Boon, G. K. (1964), Economic Choice of Human and Physical Factors in Production, North Holland Publishing Co., Cuadros 3.18 y 3.22.

Cardwell, L. A. (1978), "Technology Choice in the Mens' Leather Shoe and Cotton Spinning Industries in Brazil", citado por G. Ranis, "Technology Choice and Employment in Developing Countries: A Synthesis of Economic Growth Center Research", Economic Growth Center, Discussion Paper № 276.

Comanor, W. S. y Wilson, T. A. (1967), "Advertising, Market Structure and Performance", Review of Economic and Statistics, Vol. 49.

Dhar, E. N. y Lydall H. F. (1961), The Role of Small Enterprises in Indian Economic Development, Delhi, Asia Publishing House.

DiTullio, K. A., "The Role of Small Industries in the Political Economy of Pakistan", Syracuse, Nueva York, Disertación doctoral, Political Science Department, Syracuse University.

Gollás, M. (1975), "Reflexiones sobre la concentración económica y el crecimiento de las empresas", El Trimestre Económico, XLII (2), abriljunio.

(1978), "The Structure and Causes of Industrial Concentration in México", Economic Growth Center, Discussion Paper № 291, julio.

Guth, L. A. (1971), "Advertising and Market Structure Revisited", Journal of Industrial Economics, Vol. 19.

Kamerchen, D. R. (1968), "Market Growth and Industry Concentration", Journal of the American Statistical Association, Vol. 63.

Marsden, K. (1966), "The Role of Small Enterprises in the Industrialization of the Developing Countries”, Mimeo., Ginebra, Oficina Internacional del Trabajo.

- (1969), "Towards a Synthesis of Economic Growth and Social Justice", International Labor Review, Vol. 100, 5, noviembre, pp. 389-418.

Mehta, B. V. (1969), "Size and Capital Intensity in Indian Industry", Bulletin of Oxford University Institute of Economics and Statistics, Vol. 31, 3, agosto, pp. 189-204. 
Nelson, R. L. (1960), "Market Growth, Company Diversification and Product Concentration", Journal of the American Statistical Association, Vol. 55.

Penrose, E. (1959), The Theory of the Growth of Firms, Nueva York, Wiley.

Ranis, G. (1961), Industrial Efficiency and Economic Growth: A Case Study of Karachi, Monography No 5, Karachi, The Institute of Development Economics.

(1962), "Production Functions, Market Imperfections and Development", Economic Journal, LXXII, pp. 344-408.

Sandesara, J. C. (1966), "Scale and Technology in Indian Industry", Bulletin of Oxford University Institute of Economics and Statistics, Vol. 28, 3, agosto, pp. 181-198.

- (1969), "Size and Capital-Intensity in Indian Industry: Some Comments", Bulletin of Oxford University Institute of Economics and Statistics, Vol. 31, 4, pp. 331-334.

Savings, T. R. (1961), "Estimation of Optimum Size of Plant by the Survivor Technique", Quarterly Journal of Economics, Vol. LXXV, № 4.

Sawyer, M. C. (1971), "Concentration in British Manufacturing Industry", Oxford Economic Papers, Vol. 23.

Scherer, F. M. (1973), "The Determinants of Industrial Plant Size in Six Nations", The Review of Economics and Statistics, Vol. 55, № 2, pp. 135 145.

Shepherd, G. (1964), "Trends of Concentration in American Manufacturing Industries: 1947-1958”, Review of Economics and Statistics, Vol. 46.

Shetty, M. C. (1963), Small Scale and Household Industries in a Developing Economy, Delhi, Asia Publishing House.

Stigler, G. J. (1952), "The Theory of Olygopoly", en The Theory of Price, MacMillan.

- (1958), "The Economics of Scale", Journal of Law and Economics, Vol. 1, p. 54.

Todd, J. E. (1971), "Size of Firm and Efficiency in Colombian Manufacturing", Williams College Center for Development Economics, Research Memorandum, № 41.

Weiss, L. W. (1963), "Factors in Changing Concentration", Review of Economics And Statistics, Vol. XLVI, febrero.

(1964), "The Survival Technique and the Extent of Sub-Optimal Capacity", Journal of Political Economy, Vol. 62, junio, pp. 246-261. 